\title{
Raumordnungspolitik als Verdichtung politischer Kräfteverhältnisse
}

\author{
Michael Mießner
}

Am Beispiel der Raumordnungspolitik der Bundesrepublik Deutschland der 1970er-Jahre wird im Beitrag mithilfe der historisch-materialistischen Politikanalyse die politische Verdichtung der Kräfteverhältnisse bezüglich Raumordnung analysiert. Es wird aufgezeigt, dass das Konzept der dezentralen Konzentration mittels Entwicklungszentren und -achsen zur bestimmenden raumordnungspolitischen Strategie für die Bearbeitung der räumlich ungleichen Entwicklung in der Bundesrepublik Deutschland wurde. Vor dem Hintergrund der sich real in den 1970er-Jahren vollziehenden räumlichen Entwicklung, in Form der dezentralen Konzentration, bot es den Parteienvertreter_innen die Möglichkeit, ihre ideologischen und parteipolitischen Vorstellungen mit diesem Konzept zu verbinden. Mit dem Konzept der dezentralen Konzentration wurde eine Strategie gewählt, die die bestehende räumliche Entwicklung protegierte und ihr nichts entgegensetzte.

Ersteinreichung: 2. August 2016; Veröffentlichung online: 21. Juni 2017

An english abstract can be found at the end of the document.

\section{Einleitung}

Der Staat und seine Apparate sind - wie Nicos Poulantzas in Staatstheorie gezeigt hat - nicht starr, sondern ,als die materielle Verdichtung eines Kräfteverhältnis“ (2002: 159) zu verstehen. Diese Argumentation kann formanalytisch dahingehend erweitert werden, dass sich gesellschaftliche Kräfteverhältnisse zwar in den Staat einschreiben, sich aber nicht eins zu eins in ihn ,übersetzen'. Sie schreiben sich in den Staat nur in gebrochener Form entsprechend der - auch formbestimmten (vgl. Kannankulam 2008: 77) - strategischen Selektivität des Staates (vgl. Jessop 1990) ein. Folglich kann auch räumliche Planung und die - in diesem Aufsatz zentrale - Raumordnungspolitik nicht als von den gesellschaftlichen Verhältnissen losgelöste und neutrale Politik verstanden werden. Vielmehr ist auch sie das - widersprüchliche - Ergebnis der Verdichtung[1] gesellschaftlicher Kräfteverhältnisse.

Aufbauend auf diese Theorie wird in diesem Beitrag am Beispiel der (bundesdeutschen) Raumordnungspolitik der Frage nachgegangen, wie raumordnungspolitische Strategien im Staat verdichtet werden. Dafür wird die Methode der historisch-materialistischen Politikanalyse - wie sie in der kritischen Europaforschung für die Analyse der Kämpfe um die europäische Migrationspolitik entwickelt wurde[2] - für die raumplanerische Diskussion 
nutzbar gemacht. Diese Herangehensweise ermöglicht es aufzuzeigen, wie sich die verschiedenen raumordnungspolitischen Strategien im Staatsapparat Raumordnung zu einer verdichten. Dies soll exemplarisch anhand der politischen Auseinandersetzungen um die ,richtige' Raumordnungspolitik des Bundes in den 1970er-Jahren aufgezeigt werden.

Unter Raumordnung wird der Teil der Raumplanung verstanden, ,in dem überörtliche und fachübergreifende Entwicklungsvorstellungen formuliert und durch Programme, Pläne, Handlungskonzepte und Maßnahmen umgesetzt werden "(Sinz 2005: 863). Durch die Raumordnung werden Leitbilder für und normative Vorstellungen über die Ordnung des deutschen Staatsgebietes entwickelt, die den verschiedenen raumwirksamen Politiken des Bundes und der Länder als Orientierung dienen sollen (vgl. Krumbein et al. 2008: 16). In diesem Aufsatz wird der Raumordnungsbegriff auf die räumliche Planung des Bundes bezogen und nicht auf die Landesplanung.

Aufgrund des föderalen Aufbaus der Bundesrepublik Deutschland, in der die räumliche Planung überwiegend in der Hand der Bundesländer liegt, muss die Raumordnungspolitik des Bundes auf einer vergleichsweise allgemeinen Ebene agieren und kann selbst allenfalls großräumige Handlungsvorschläge unterbreiten. Sie besitzt aber keine verbindliche Richtlinienkompetenz gegenüber den Bundesländern oder Bundesfachpolitiken (vgl. Durner/Greiving/ Reitzig 2011). Die Bundesraumordnungspolitik - die in diesem Aufsatz in Abgrenzung zu anderen staatlichen Apparaten und Zweigen als Staatsapparat Raumordnung bezeichnet wird - verfügt dementsprechend über wenige Kompetenzen, mit denen sie die räumliche Entwicklung direkt beeinflussen kann. Dieser Staatsapparat ist vielmehr darauf angewiesen, dass die von ihm vorgeschlagenen Handlungsstrategien durch andere staatliche Apparate[3] umgesetzt werden. Anhand dieser Ausführungen wird gleichzeitig deutlich, dass die räumliche Entwicklung in der Bundesrepublik Deutschland durch unterschiedliche staatliche Apparate und Zweige beeinflusst wird. Neben dem Wirtschaftsministerium sind hier unter anderem die Ministerien für die Infrastrukturentwicklung und Landwirtschaft zu nennen.

Dass Raumordnung in der Bundesrepublik Deutschland auf nationalstaatlicher Ebene angesiedelt ist, stellt im Vergleich zu anderen westlichen Wohlfahrtsstaaten eine Besonderheit dar (vgl. Brenner 2004). Dieser besondere Staatsapparat, der von zentralstaatlicher Seite die räumliche Entwicklung zu bearbeiten beansprucht (vgl. ebd.: 142), ist daher der Untersuchungsgegenstand des vorliegenden Aufsatzes. Nicht untersucht wird dagegen die Landes- und Regionalplanung.

Die ,richtigen' Problematisierungen und Leitlinien der räumlichen Entwicklung handeln - aufgrund der vergleichsweise hohen Abstraktionsebene - überwiegend Politiker_innen, Ministeriumsvertreter_innen, Vertreter_ innen der Landesplanung der Bundesländer sowie Raumwissenschaftler_ innen aus. Selten sind dagegen außerparlamentarische Stimmen - wie die der Gewerkschaften oder Wirtschaftsverbände - in den Diskussionen zu hören. Die Auswirkungen raumordnungspolitischer Entscheidungen wiederum sind im Alltag der Bürger_innen selbst kaum spürbar. Diese abstrakten Leitlinien werden höchstens durch ihre landespolitische, regional- und stadtplanerische Umsetzung wahrnehmbar. Insofern handelt es sich bei den politischen Aushandlungsprozessen bezüglich Raumordnungspolitik 
des Bundes weniger um alltägliche politische Praxen (von Bürger_innen), als um (raumordnungs-)politische und wissenschaftliche.

Im Folgenden wird zunächst ,Staat' im Anschluss an die historischmaterialistische Theoriebildung gefasst und mithin als materielle Verdichtung gesellschaftlicher Kräfteverhältnisse konzeptualisiert (Abschnitt 2). Darauf aufbauend wird dieses Staatsverständnis für die Konzeptualisierung der Regulation räumlicher Verhältnisse mittels Raumordnung im Rahmen des state spatial process (vgl. Brenner 2004) nutzbar gemacht (Abschnitt 3). Im darauf folgenden Abschnitt 4 wird die historisch-materialistische Politikanalyse vorgestellt. Anschließend wird mithilfe dieser Methode die Raumordnungspolitik in der Bundesrepublik Deutschland in den 1970er-Jahren analysiert (Abschnitt 5). Der Beitrag wird mit einem Fazit abgerundet.

\section{Der Staat als materielle Verdichtung gesellschaftlicher Kräfteverhältnisse}

In der materialistischen Staatstheorie gilt der Staat als wichtiger, ,institutioneller Knotenpunkt" (Hirsch 1994: 192; Herv. i. O.) für die Regulation kapitalistischer Verhältnisse. Dabei werden „Staat und Gesellschaft nicht als dualistisch voneinander getrennte Sphären“ (Wissel/Wöhl 2008: 9) verstanden. Der Staat gilt vielmehr als „soziales Verhältnis“(ebd.). In einer formanalytischen Begründung des Staates wird argumentiert, dass sich die Subjekte - die sich im Austauschprozess als Warenbesitzer_innen gegenüberstehen -als freie und gleiche Subjekte zueinander verhielten. Sie würden so zu „Träger[n] von Rechten“ (Paschukanis 1929: 90). Der ,Garant‘ (vgl. Marx 1962: 99) dieser Rechtsverhältnisse sei der Staat. Damit sei dieser eine notwendige Voraussetzung kapitalistischer Vergesellschaftung. Die formanalytische Bestimmung des Staates dürfe jedoch nicht als funktionalistische missverstanden werden (vgl. Hirsch/Kannankulam 2006: 77, Kannankulam 2008: 51). Es sei vielmehr so, dass die „Bestimmung der politischen Form auf dieser hohen Abstraktionsebene [...] keine Aussagen über die institutionelle Struktur und die Funktionsweise“ (Hirsch/Kannankulam 2006: 77) des Staates zulasse.

Für die Erklärung konkreter staatlicher Praxis ist in der historisch-materialistischen Theorie die Staatstheorie von Nicos Poulantzas (2002) ein zentraler Bezugspunkt. Für ihn ist der Staat der zentrale Ort, an dem sich die politischen Klassen- und Fraktionskämpfe manifestierten. Diese Auseinandersetzungen spiegelten die gesellschaftlichen Klassenkämpfe jedoch nicht direkt wider. Vielmehr seien sie durch das „materielle Gerüst“ (ebd.: 161) des Staates geprägt. Zwar hätten Veränderungen „des Kräfteverhältnisses [...] immer Auswirkungen innerhalb des Staates“ (ebd.: 162), diese übertrügen „,sich jedoch nicht direkt und unmittelbar“ (ebd.), sondern passten „,sich der Materialität der verschiedenen Apparate an und kristallisier[en] sich im Staat nur in gebrochener und differenzierter, den Apparaten entsprechender Form“ (ebd.). Die verschiedenen Staatsapparate, wie „Exekutive und Parlament, Armee, Justizbehörden, verschiedene Ministerien, kommunale Apparate“ (ebd.), aber auch die Raumordnung, seien in diesem Sinne der „Sitz der Macht und spezielle Vertretung irgendeiner Fraktion des Blocks an der Macht oder eines konflikthaften Bündnisses dieser Fraktionen gegen andere" (ebd.). Staatsapparate seien demnach Orte, an denen spezifische Klasseninteressen 
oder Zusammenschlüsse von Sonderinteressen einen institutionellen Ausdruck erhielten. Gleichzeitig sei der Staat - laut Bob Jessop - durch eine ,strategische Selektivität' (1990: 9) gekennzeichnet. Diese strategische Selektivität besitzt der Staat, da er - aufgrund seiner Struktur und darin eingeschriebener Kräfteverhältnisse - bestimmte gesellschaftliche Kräfte privilegiert und ihnen den Zugang zum Staat erleichtert.

\section{Regulation räumlicher Verhältnisse mittels Raumordnung}

Das Ziel der Raumordnung ist es, räumliche Entwicklungen so zu fördern, dass sie den normativen Vorstellungen von der Ordnung des deutschen Staatsgebietes entsprechen. Folglich stellt die räumliche Entwicklung die zentrale Grundlage und gleichzeitig den zu bearbeitenden Gegenstand der Raumordnungspolitik dar (vgl. Mießner 2015).

Wie David Harvey (2006) und Neil Smith (1984) in ihren Arbeiten gezeigt haben, ist die räumliche Entwicklung unter kapitalistischen Verhältnissen stets durch uneven development gekennzeichnet. Die Widersprüche kapitalistischer Vergesellschaftung drücken sich einerseits räumlich aus. Andererseits ist die ungleiche räumliche Entwicklung auch Mittel der Kapitalakkumulation: Ist eine Region beispielsweise ökonomisch unterentwickelt - weil aus ihr Kapital abgezogen wurde, um es in anderen Regionen zu investieren -, kann sie wiederum sehr profitabel und zum Ausgangspunkt neuer Kapitalakkumulation werden (vgl. Smith 1984: 149).

Ziel des Staates ist es, im Rahmen des state spatial process (Brenner 2004) in die räumliche Entwicklung zu intervenieren, ,to establish a 'structured coherence' or 'spatial fix' for capitalist growth" (Brenner 2003: 205). Der Raumordnungspolitik - als Teil des state spatial process - geht es um die Etablierung eines „spatial fix anchored firmly within the national territorial matrix“ (Brenner 1997: 286). Dies bedeutet jedoch nicht, dass sie die bestehenden räumlichen Ungleichheiten abmildern will. Da uneven development Ausdruck und Mittel kapitalistischer Gesellschaften ist, können zu große regionale Disparitäten zwar hinderlich sein. Zugleich ist ein gewisses Ausmaß räumlicher Ungleichheiten gerade zweckdienlich.

Diese formanalytische Betrachtung der Raumordnung sagt noch wenig über ihre konkrete Ausgestaltung aus. Diese ist von den gesellschaftspolitischen Kräfteverhältnissen abhängig, die sich in der Raumordnung verdichten. Insofern ist Raumordnung als „condensation of social relations“ (Holgersen 2013: 5) zu verstehen.

Im Folgenden soll mit der historisch-materialistischen Politikanalyse eine Methode vorgestellt werden, mithilfe derer die Verdichtung raumordnungspolitischer Kräfteverhältnisse nachgezeichnet und erklärt werden kann. Anschließend wird dargestellt, wie die raumordnungspolitischen Aushandlungsprozesse in den 1970er-Jahren verliefen.

\section{Historisch-materialistische Politikanalyse und critical discourse analysis}

Zur Operationalisierung der materialistischen Staatstheorie wurde die historisch-materialistische Politikanalyse entwickelt. Sie ist zum einen in 
Auseinandersetzung mit den policy-Forschungen entstanden, deren Vertreter innen von einem vereinfachten Staatsverständnis ausgehen und Machtverhältnisse innerhalb des Staates ausblenden (vgl. Brand 2013). Zum anderen grenzen sich historische Materialist_innen von strukturalistischeren Ansätzen in der materialistischen Staatstheorie ab, die politische Strategien wiederum „oft in einer funktionalistischen Weise als ,Ergebnis' dominanter sozialer Verhältnisse, insbesondere der Klassenverhältnisse“ (ebd.: 425) konzeptualisieren.

Die historisch-materialistische Politikanalyse wurde zur Untersuchung der Formierung des ,Staatsprojektes Europa“ und der europäischen Migrationspolitik entwickelt (vgl. beispielsweise Forschungsgruppe Staatsprojekt Europa 2014, Wissel 2015). Ziel war es, die Verdichtung der Kräfteverhältnisse in den EU-Politiken nachzuvollziehen. Abhängig von der Fragestellung stehen „unterschiedliche gesellschaftliche Bereiche mit [...] spezifischen Selektivitäten und Eigenlogiken im Mittelpunkt einer Untersuchung“ (Buckel et al. 2014: 53), weshalb die im Rahmen der historisch-materialistischen Politikanalyse vorgeschlagenen Analyseschritte - Kontextanalyse, Akteursanalyse und Prozessanalyse - für die jeweiligen Forschungen zu präzisieren sind. Im Folgenden werden diese drei Analyseschritte vorgestellt und für die Untersuchung der Raumordnungspolitik nutzbar gemacht.

\subsection{Kontextanalyse}

Im Rahmen der Kontextanalyse geht es darum, „den historisch-dynamischen und strukturellen Kontext des jeweils untersuchten Konfliktes“ (Buckel et al. 2014: 54) zu analysieren. Es gilt, die historische Situation des Konfliktes zu rekonstruieren (vgl. ebd.).

In diesem Aufsatz wird erstens der größere ökonomische und insbesondere politische Kontext der Raumordnungspolitik der 1970er Jahre in der Bundesrepublik Deutschland dargestellt. Zweitens werden die räumlichen Implikationen dieser gesellschaftlichen Entwicklungen herausgearbeitet - schließlich sind dies die Entwicklungen, die die Raumordnungspolitik beeinflussen möchte.

\subsection{Akteursanalyse}

Im Zuge der Akteursanalyse gilt es zu untersuchen, welche gesellschaftlichen und politischen Akteur_innen in einer konkreten historischen Situation um die Lösung eines bestimmten gesellschaftlichen Problems ringen (vgl. Kannankulam/Georgi 2014: 63ff., Wissel 2015: 65f.). Weiterhin geht es darum, die in einem Konflikt „präsenten konfligierenden Strategien“ (Buckel et al. 2014: 55) der unterschiedlichen Akteur_innen und Kräfte herauszuarbeiten. Dazu sind eine genaue Kenntnis der Sekundärliteratur sowie sogenannte,Debattenanalysen' (Kannankulam/Georgi 2012: 38), die auf einer intensiven Auswertung der stattfindenden Diskussionen fußen, notwendig.

Im Rahmen der Akteursanalyse gilt es daher erstens im Zuge der ,Situationsanalyse ' darzustellen, was im Diskurs problematisiert und was als Ursache des Problems identifiziert wird (vgl. Buckel et al. 2014: 56). Zweitens ist das strategische Ziel aufzuzeigen, das die jeweiligen politischen Kräfte im untersuchten Konflikt verfolgen (vgl. ebd.: 56f.). 


\subsection{Prozessanalyse}

In der Prozessanalyse ist der untersuchte „Konflikt chronologisch, in seiner mehrere Phasen durchlaufenden historischen Dynamik“ (Kannankulam/ Georgi 2012: 39) zu analysieren. Um die „komplexen Kampfprozesse, in denen sich der Konflikt" (Buckel et al. 2014: 58) entwickelt hat, verstehen zu können, sind die Kontext- und Akteursanalyse zu kombinieren (ebd.). Je nach Fragestellung verschiebt sich der Analyseschwerpunkt. In diesem Aufsatze soll die Verdichtung der politischen Kräfteverhältnisse zu raumordnungspolitischen Strategien nachgezeichnet werden. Daher werden die im Staatsapparat Raumordnung problematisierten räumlichen sowie gesellschaftlichen Entwicklungen und die für diese Probleme vorgeschlagenen Handlungsstrategien dargestellt. Auf diese Weise kann gezeigt werden, wie die verschiedenen raumordnungspolitischen Projekte im Staatsapparat Raumordnung zu einer - einheitlichen - Strategie verdichtet wurden.

\subsection{Critical discourse analysis}

Die vorgestellte Akteurs- und Prozessanalyse stellt eine Heuristik dar, die es wie dieVertreter_innen dieser Methode explizit betonen (vgl. Brand 2013:437, Buckel et al. 2014: 53ff.) - für den jeweiligen Untersuchungsgegenstand zu präzisieren gilt. Hierfür bietet sich die Methode der critical discourse analysis (vgl. beispielsweise Fairclough 2010, Wodak/Meyer 2001) an, eine Methode, die bereits für die materialistische Staatstheorie nutzbar gemacht wurde (vgl. Fairclough 2010: 375ff., Jessop 2008: 225ff.). Sie ermöglicht diese beiden Analyseschritte empirisch zu operationalisieren. Da das Untersuchungsmaterial in Form von Texten (Berichte, Gesetze, Protokolle) vorlag (siehe Abschnitt 5), ist die critical discourse analysis für die Operationalisierung geeignet, wurde sie doch genau dafür entwickelt. Hier gilt es insbesondere, das Material entsprechend der Problematisierungen (vgl. Fairclough 2010: 235f., Fairclough/ Pardoe/Szerszynski 2010: 413ff.) zu strukturieren. Darüber hinaus wurden konfliktbezogene Aussagen, Handlungsvorschläge und Taktiken der einzelnen politischen Kräfte analysiert.

\section{Aushandlungsprozesse um die Raumordnungspolitik in den 1970er-Jahren}

Im Folgenden soll das analytische Potential der historisch-materialistischen Politikanalyse anhand der bundespolitischen Auseinandersetzungen um die anzustrebende Raumordnungspolitik in den 1970er-Jahren aufgezeigt werden. Dieser Zeitraum gilt gemeinhin als eine Phase der Raumordnungspolitik, in der zentralstaatliche Raumsteuerung zugunsten endogener Entwicklungsstrategien in den Hintergrund trat (vgl. Blotevogel 2011, Brenner 1997; 2004, Heeg 2001, Mießner 2009).

Untersucht wurden unter anderem Berichte, Gesetzesvorlagen und Stellungnahmen, die von Bundesministerien oder -behörden veröffentlicht wurden. Dazu zählen beispielsweise die Raumordnungsberichte, das Bundesraumordnungsprogramm oder die Tätigkeitsberichte des für Raumordnung zuständigen Bundestagsausschusses. Weiterhin wurden die 
Diskussionsprotokolle des Bundestags und seiner Ausschüsse - wie der Ausschuss für Raumordnung, Bauwesen und Städtebau sowie der Unterausschuss Regionale Wirtschaftspolitik des Ausschusses für Wirtschaft und Technik - untersucht. Außerdem waren die Diskussionsprotokolle des Bundesrates und des Bundesratsausschusses für Städtebau, Wohnungswesen und Raumordnung - die sich ebenfalls mit raumordnungspolitischen Themen beschäftigten - Grundlage der Analyse. In die Untersuchung wurden darüber hinaus die Beschlüsse der Ministerkonferenz für Raumordnung und die Stellungnahmen des Beirates für Raumordnung einbezogen. Damit konzentriert sich das Untersuchungsmaterial auf die Auseinandersetzungen innerhalb des Staatsapparates Raumordnung und auf Diskussionen zwischen den Staatsapparaten, die an der Raumordnungspolitik mitwirken, insbesondere auf die Auseinandersetzung zwischen den im Bundestag vertretenen Parteien. Die methodische Auswertung eines noch größeren Untersuchungskorpus war mit den gegebenen Forschungsressourcen nicht möglich. Dies dürfte - neben dem hohen Abstraktionslevel, auf dem die Raumordnung agiert ein weiterer Grund sein, warum im untersuchten Diskurs keine Stimmen von Bürger_innen oder Interessensvertretungen außerparlamentarischer Gruppierungen ausgemacht werden konnten.

Der Untersuchungszeitraum wurde auf die Jahre 1973 bis 1978 eingegrenzt. Im Folgenden werden die Ergebnisse der einzelnen Analyseschritte gemäß der historisch-materialistischen Politikanalyse dargestellt und aufgezeigt, wie die Verdichtung der politischen Kräfteverhältnisse bezüglich Raumordnung in der Bundesrepublik Deutschland in den 1970er-Jahren vonstattenging.

\subsection{Kontextanalyse: ökonomische Verwerfungen}

In der Bundesrepublik Deutschland ereignete sich in den Jahren 1966 und 1967 die erste Rezession der Nachkriegszeit (vgl. SVR 1967: 1). Diese war jedoch schnell wieder überwunden (vgl. Altvater/Hübner 1988: 17f.). Gleichzeitig erfolgte ein Regierungswechsel. So wurde die schwarz-gelbe Regierung 1966 von der Großen Koalition aus CDU/CSU und SPD abgelöst, die 1969 wiederum einem sozialliberalen Bündnis weichen musste. Diese Regierungswechsel gelten gemeinhin als wirtschafts- und sozialpolitische Richtungsänderung, weg von stärker ordoliberalen Vorstellungen hin zur keynesianischen Globalsteuerung unter Einbeziehung der Gewerkschaften (vgl. Abelshauser 2004: 412). Der neue SPD-Wirtschaftsminister Karl Schiller stand exemplarisch für diesen Kurs ein (vgl. zum Beispiel Schiller 1964). Mit ihm war gleichsam ein politischer Richtungswechsel hin zu einer stärker sozial- und ausgleichsorientierten Wirtschafts- und Sozialpolitik verbunden (vgl. Abelshauser 2004).

Räumlich hatte sich die wirtschaftliche Entwicklung bis Mitte der 1960erJahre in Form der dezentralen Konzentration (vgl. Väth 1980: 69ff.)[4] vollzogen. Die großen Wachstumsraten, die die deutsche Wirtschaft mithilfe der fordistischen Massenproduktion erzielte, führten zu einer räumlichen Konzentration der Industrieproduktion und damit auch der Arbeitskräfte sowie der Bevölkerung. Diese Entwicklung ging zu Lasten der ländlichen Räume und kleinen Gemeinden. Da die Arbeitskräfte in den Ballungsräumen aufgrund steigender Produktion knapp wurden, dezentralisierte sich die Produktion zunehmend und erreichte zuerst die Zentren des Umlandes und 
später die der weiteren Peripherien (vgl. BMWSR 1963: 16ff., Bundesregierung 1966: 12ff., SVR 1965). Diese dezentrale Konzentration wirkte aus einer großräumigen Perspektive ausgleichend: Es hatte den Anschein, als würden mit dem wirtschaftlichen Wachstum die wirtschaftlichen Strukturprobleme in den ländlichen und peripheren Regionen en passant behoben werden. Die damit verbundenen innerregionalen Disparitäten gerieten aus dieser großräumigen Perspektive allerdings aus dem Blick.

Die Krise von 1966 und 1967 traf neben den „Deglomerationsräume[n] und [...] Agglomerationen, die durch Monostrukturen im Montanbereich gekennzeichnet waren (Rhein-Ruhr, Saar)“ (Jacob 1979: 27), vor allem strukturschwache Peripherien. Diese hatten noch bis 1966 von sehr „konjunkturreagib[len]“ (Heeg 2001: 127) Zweigwerks- und kleinbetrieblichen Neugründungen profitiert (vgl. Jacob 1979: 27ff). In der folgenden Aufschwungphase bis Mitte der 1970er-Jahre setzte sich die dezentrale Konzentration fort: Besonders die Verdichtungsräume und - aufgrund von erneuten Zweigwerksgründungen - deren Umland sowie die zentralen Orte profitierten vom Wirtschaftswachstum (vgl. ebd.: 3off.). Die ländlichen und strukturschwachen Regionen blieben - abgesehen von deren Zentren - hingegen hinter der allgemeinen Entwicklung zurück (vgl. ebd.). Die innerregionalen Disparitäten verstärkten sich erneut.

Anfang der 1970er-Jahre wurden das Bretton-Woods-System und damit die Golddeckung des Dollars sowie das System fixer Wechselkurse aufgegeben. Die mit dem Bretton-Woods-System verbundene Unterbewertung der D-Mark hatte in den 1960er-Jahren ein exportgetragenes Wirtschaftswachstum bewirkt (vgl. Kannankulam 2008: 169). Diese „gewaltigen Fehlleitung[en] von Kapital“(Polster/Voy 1991: 197ff.) mussten in den 1970er-Jahren kompensiert werden. Weil ab Mitte der 1970er-Jahre auch alle anderen Industrienationen von der Rezession betroffen waren (vgl. Altvater/Hübner 1988: 20), wurde die exportgetriebene Wirtschaftsentwicklung der Bundesrepublik Deutschland vor zusätzliche Probleme gestellt. Diese Entwicklungen wurden 1973 durch den Ölpreisschock noch verstärkt. Im Jahr 1975 sank das Bruttosozialprodukt der Bundesrepublik und die Arbeitslosigkeit stieg dauerhaft auf über 4,6 Prozent (vgl. Prollius 2006: 185ff., SVR 1978: 64).

Trotz des „Sonderprogramms zur regionalen und lokalen Abstützung der Beschäftigung“ (in Höhe von 950 Millionen D-Mark) und des „Programms zur Förderung von Beschäftigung und Wachstum bei Stabilität“ (in Höhe von 1,13 Milliarden DM) erholte sich die deutsche Wirtschaft kaum. In den folgenden Jahren schwenkte die sozialliberale Regierung unter dem neuen Kanzler Helmut Schmidt auf Austeritätspolitik um (vgl. Altvater/Hübner 1988: 25). Diese war zunächst zögerlich, später vehement von den,Wirtschaftsweisen' gefordert worden (vgl. SVR 1975: 16ff.; 1976: 139ff.). Beispielsweise wurde 1975 ein Haushaltsstrukturgesetz verabschiedet, in dessen Rahmen Sozialausgaben gekürzt wurden (vgl. ebd.). Solchen und anderen Spar- und Konsolidierungsbemühungen fielen „hauptsächlich die Investitionen der Gemeinden zum Opfer“ (Polster/Voy 1991: 208), womit vor allem die „Kernbereiche der öffentlichen materiellen Infrastrukturen in Verkehr, Ver- und Entsorgungsfunktionen, Wohnungs- und Städtebau“ (ebd.) betroffen waren.

Von diesen Entwicklungen profiterten bis Mitte der 1970er-Jahre vor allem die Agglomerationsräume wirtschaftlich, politisch und demografisch 
(vgl. Gatzweiler/Runge 1978: 673ff.). Gleichzeitig entwickelten sich die Regionen mit Verdichtungsansätzen oder jene mit einem regionalen Zentrum lediglich durchschnittlich, während die ländlichen Regionen zurückblieben (vgl. ebd.). In der Infrastrukturversorgung wurden bis Mitte der 1970er-Jahre erhebliche Fortschritte in den Peripherien erreicht, weshalb „Entwicklungsimpulse durch vermehrte öffentliche Investitionen auf diesem Gebiet kaum mehr induzierbar“ (Väth 1979: 62) waren. Außerdem gingen die unternehmerischen Investitionen nach und nach zurück, weshalb die „räumlich disponible Investitionsmasse“ (ebd.) schrumpfte. Der „klassische Ansatzhebel der regionalen Wirtschaftspolitik, die als regionale Kapitalmobilisierungsstrategie konzeptionalisiert war" (ebd.), konnte somit kaum noch Wirkung entfalten. Außerdem wurden die betrieblichen Investitionen zunehmend für Rationalisierungen eingesetzt, sodass einerseits Neuansiedlungen von Unternehmen die Auswirkungen von Betriebsstilllegungen nicht mehr kompensieren konnten (vgl. Jacob 1979: 43). Andererseits waren die Arbeitskraftreserven als „natürliche[r] Standortvorteil“ (Väth 1979: 63) peripherer Regionen nicht mehr für unternehmerische Standortentscheidungen ausschlaggebend. Eine weitere dezentrale Konzentration fand somit nicht mehr statt. Bestehende Standorte blieben zwar erhalten, neue wurden jedoch nicht mehr geschaffen.

Diese Entwicklungen wurden gegen Ende der 1970er-Jahre durch ein ,Nord-Süd-Gefälle überlagert, das heißt die nördlichen und südlichen Agglomerationsräume entwickelten sich zunehmend auseinander (vgl. Friedrichs/Häußermann/Siebel 1986). Während sich im Norden und Westen Deutschlands ältere Industrien fordistischer Massenproduktion befanden, waren die prosperierenden und diversifizierten Agglomerationsräume im Süden durch High-Tech-Industrien geprägt (Brenner 1997: 288f.).

\subsection{Akteursanalyse: ökonomischer Strukturbruch und großräumige Disparitäten als Problem}

Im untersuchten raumordnungspolitischen Diskurs wurden die zunehmenden Disparitäten zwischen Verdichtungsräumen und ländlichen Regionen als wichtiges Problem erkannt und diskutiert. Die in diesem und im nächsten Abschnitt herangezogenen Aussagen von Parlamentarier_innen stellen dabei keine Einzelmeinungen dar, sondern sind charakteristisch für die Argumentation der jeweiligen Partei.

Die während der Untersuchungszeit im Bundestag vertretenen Parteien bewerteten die Disparitäten zwischen Verdichtungsräumen und ländlichen Regionen unterschiedlich. Ursächlich hierfür waren einerseits die unterschiedlichen Wähler_innenklientels, andererseits ideologische Vorstellungen. Die CDU/CSU-Fraktion, die stärker in den ländlichen Regionen verankert war, war nicht daran interessiert, den Verstädterungsprozess zu unterstützen. Sie wollte die Entwicklung in den ländlichen Regionen fördern. Ideologisch hatte sich die CDU/CSU-Fraktion seit den 1950er-Jahren in eine ,Richtung (Väth 1980: 154) entwickelt, die in einer ,allgemeinen Stadtfeindlichkeit und einer Eigenheim- und Grundbesitzideologie“ (ebd.) verwurzelt war. Weiterhin bestand innerhalb der CDU/CSU-Fraktion die „unterschwellige Befürchtung vor einer zu hohen Konzentration von Arbeitskräften [...], der 
allgemein sozial destabilisierende Effekte zugesprochen wurden“ (ebd.). Eine solche Vorstellung deckte sich zusätzlich mit der ,neuen Ballungskritik', die aufgrund der fortschreitenden Verstädterung immer mehr Aufwind bekam (vgl. Leendertz 2008: 317ff.).

Entsprechend dieser Konstellation unterstellte der Abgeordnete FriedrichAdolf Jahn (CDU/CSU-Fraktion) der SPD, sie wolle einzig die Verdichtung fördern:

„Die Qualität des Lebens besteht nicht in einer Quantität der Verdichtung, [...] vielmehr in einer Ausgewogenheit zwischen geordneter Verdichtung und den ländlichen Räumen.“ (1974: 7929)

Eine solche Ausgewogenheit, mithilfe derer auch die ländliche Entwicklung gefördert werden sollte, sahen die CDU/CSU-Fraktionsabgeordneten in Gefahr, auch wenn ihnen klar war, dass in der Tat Unterschiede zwischen den beiden Gebietskategorien bestanden und weiterhin bestehen würden.

Auch die SPD sah das Wachstum der Verdichtungsräume als Problem an, stand diesem Phänomen aber weniger kritisch gegenüber. Ursächlich dafür dürfte sein, dass die Verdichtungsräume „traditionell höhere Wähleranteile der SPD auf[wiesen] als ländliche und dezentralisierte Gebiete“ (Väth 1980: 154). Der Fokus der SPD lag vor allem auf den Ursachen des Verdichtungsprozesses: Verantwortlich für diese Entwicklungen seien die Lohnangleichungen zwischen ländlichen Regionen und Verdichtungsräumen (vgl. Immer 1975a: 6), die jüngste wirtschaftliche Krise (vgl. Schmitt-Vockenhausen 1974: 7951) sowie das Standortverhalten der Industrien, das sich immer öfter an den Fühlungsvorteilen in den Ballungsregionen orientiere (vgl. Immer 1975a: 6).

Die FDP problematisierte die Disparitäten zwischen Verdichtungsräumen und ländlichen Regionen weniger. Sie galten ihr vielmehr als Ausdruck einer freiheitlichen Entwicklung. Folglich war für sie der „Gegensatz von Stadt und Land [...] nicht auflösbar“ (Engelhard 1974: 7949) - alles andere sei eine „Illusion“ (Böger 1976: 16755):
„Aber umgekehrt [entgegen der Ansicht, dass die Wanderung in die Großstädte ein Problem sei; Anmerk. d. A.] muß man die Entwicklung immer auch auf dem Hintergrunde der historischen Erfahrungstatsache sehen, daß sich größere Städte in ihrem Bestand schon immer nur erhalten oder gar wachsen konnten, weil sie Zuzug vom Land hatten. Das war lange vor der ersten industriellen Revolution so, das war schon im Mittelalter so.“ (Engelhard 1978: 8633)

Diese Problematisierung des Stadt-Land-Gegensatzes macht zwei Dinge deutlich. Zum einen ist die freiheitliche Entwicklung, in der Argumentation der FDP, durch eine disparate räumliche Entwicklung gekennzeichnet. Anhand ihrer Argumentation wird weiterhin ersichtlich, dass sie die ungleiche räumliche Entwicklung naturalisierte, indem sie ihr absprach eine spezifisch historische, kapitalistische Entwicklung zu sein. Folgerichtig verweist die Aussage des Abgeordneten Böger darauf, dass man dieser dichotomen räumlichen Entwicklung nichts entgegensetzen könne.

Eine etwas andere Position nahm der Beirat für Raumordnung ein. Er fungierte als Beratungsgremium für das Bundesministerium, welches für 
Belange der Raumordnung zuständige war, und bestand aus Sachverständigen, Wissenschaftler_innen sowie Vertreter_innen kommunaler Verbände. Die sich verstärkenden Disparitäten zwischen Verdichtungsräumen und ländlichen Regionen stellten für den Beirat ein Problem dar. Aus seiner Sicht wirkte der mit der disparaten Entwicklung verbundene räumliche Konzentrationsprozess „,in zweifacher Hinsicht polarisierend“ (Beirat für Raumordnung 1972b: 163): ,[E]inmal in [B] ezug auf die räumlichen Strukturen, d. h. den Verdichtungsräumen stehen immer deutlicher Entleerungsräume gegenüber“ (ebd.), und darüber hinaus „in [B] ezug auf die spezifischen Standortqualitäten der Verdichtungsknoten bzw. der Verdichtungsachsen“ (ebd.).

Im Bundesraumordnungsprogramm, das die Bundesregierung veröffentlichte, wurde eine sehr großräumige Perspektive gewählt. Hier wurde zwischen Räumen mit einer schwachen Siedlungsstruktur und geringen Entwicklungsmöglichkeiten einerseits und Regionen mit hoher Verdichtung andererseits unterschieden (vgl. Bundesregierung 1975b: 20). Laut Raumordnungsprogramm seien jedoch innerregionale Disparitäten - wie beispielsweise in den Räumen Rhein-Ruhr und Rhein-Main-Neckar - „nicht negativ zu beurteilen“ (ebd.: 17), solange diese „auf einer räumlich-funktionalen Aufgabenteilung beruhen" (ebd.).

Trotz dieser Differenzen im raumordnungspolitischen Diskurs gab es auch Gemeinsamkeiten in der Problemwahrnehmung: Die - mit den gesellschaftlichen Entwicklungen der 1970er-Jahren einhergehende - Akzentuierung der räumlichen Entwicklung wurde als grundlegende wirtschaftliche Umstrukturierung verhandelt, welche das raumwirksame Entwicklungspotential und damit die Steuerungsmöglichkeiten der Raumordnung stark einschränkten. So seien die bisherigen Entwicklungen durch Bevölkerungszunahme, stetiges Wirtschaftswachstum, günstige und ausreichende Energie sowie abnehmende natürliche Ressourcen gekennzeichnet gewesen (vgl. Bundesregierung 1975a: 28). Diese ,Faktoren' hätten sich nun ,gewandelt' und zukünftig dürften für die „,äumliche Entwicklung andere, regional teilweise unterschiedliche Entwicklungstendenzen maßgebend sein, bzw. bisherige Entwicklungen sich stärker akzentuieren“ (ebd.: 28f.). Dies sei ein Problem, weil das „raumwirksame Entwicklungspotential“ (ebd.: 29) zukünftig „wesentlich knapper“ (ebd.) sein werde. Unter dem Begriff des ,raumwirksamen Entwicklungspotentials‘ wurden verschiedene die Raumordnung einschränkende Entwicklungen argumentativ zusammengeführt:

1. Der sektorale Strukturwandel begrenze das raumwirksame Entwicklungspotential, weil einerseits der relevanteste ländliche Wirtschaftszweig (der Agrarsektor) an Wichtigkeit verliere (vgl. ebd.). Andererseits gehe die Bedeutung des sekundären Sektors leicht zurück, was Entwicklungsnachteile für bisherige Industrieregionen zeitige (vgl. ebd.: 64).

2. Parlamentarier_innen der CDU/CSU- und der SPD-Fraktion betonten weiterhin das veränderte Standortverhalten der Industrie. Die Standortwahl von Industriebetrieben sei laut CDU/CSU kaum noch staatlich beeinflussbar (vgl. Nordlohne 1973: 39), weil beispielsweise „Fühlungsvorteile, wie sie nur der Ballungsraum bietet, ausgenutzt werden“ (Immer 1975a: 5), wie die SPD betonte.

3. Weiterhin begrenzten die zunehmenden internationalen Verflechtungen 
den „nationalen Gestaltungsspielraum“(Bundesregierung 1975a: 10), weil die Verlagerung von Industriestandorten ins Ausland zu verhindern sei.

4. Die Zunahme der Unternehmensgrößen und die damit verbundene räumliche Maßstabsvergrößerung begrenze das raumwirksame Entwicklungspotential, weil sich so die Einzugsbereiche öffentlicher Einrichtungen „ständig vergrößern“ (Bundesregierung 1972: 28, vgl. Beirat für Raumordnung 1972b: 166f.). Somit verstärke sich der räumliche Konzentrationsprozess und schwäche sich nicht ab.

Außerdem habe der wirtschaftliche Umstrukturierungsprozess die ländlichen Räume besonders getroffen, weil hier „[e]ine große Zahl von Filialbetrieben und Zulieferfirmen [...] ihre Tore für immer geschlossen" (Sauter 1978: 8630) hätten, argumentierten Vertreter_innen der CDU/CSU-Fraktion. Laut SPD sei die Problematik der Konzentrationsförderung erst jüngst ,in dieser Schärfe deutlich geworden“ (Schmitt-Vockenhausen 1974: 7951).

\subsection{Prozessanalyse: dezentrale Konzentration als Kompromiss}

Das übergeordnete Ziel der Raumordnungspolitik der 1970er-Jahre war der „Abbau bestehender großräumiger Unterschiede“ (Bundesregierung 1974: 2). Das gesamtwirtschaftliche Wachstum sollte bei möglichst geringen sozialen Kosten gefördert werden. Dieses Ziel beinhaltete jedoch Widersprüche:

„Das Ziel, großräumige Disparitäten abzubauen, kann im Einzelfall zu Konflikten führen. Ein solcher Zielkonflikt kann auftreten zwischen dem Streben nach einem möglichst hohen gesamtwirtschaftlichen Wachstum, das sich vor allem in den Räumen mit hoher Verdichtung konzentrieren würde, und dem Ziel, insbesondere in ländlich geprägten Gebieten eine ausgewogene Wirtschaftsstruktur aufzubauen. Die Erwerbstätigen würden zwar durch Abwanderung in die stark verdichteten Gebiete dort kurzfristig einen größeren Beitrag zum Bruttoinlandsprodukt erbringen; dennoch müssen die Voraussetzungen für ihr Verbleiben in den ländlich geprägten Gebieten geschaffen werden, zumal dadurch - längerfristig gesehen - ein höherer sozialer Nutzen und damit zugleich ein Beitrag zur Verbesserung der Lebensqualität für alle Bürger erzielt werden können.“ (Bundesregierung 1975b: 8)

Das wichtigste - aber auch umstrittenste - Mittel, mit dem dieses übergeordnete raumordnungspolitische Ziel erreicht werden sollte, war die dezentrale Konzentration. Mit diesem Konzept konnte die reale räumliche Entwicklung, die genau diese Form angenommen hatte (vgl. Abschnitt 5.1), unterstützt werden. Darüber hinaus - und das soll im Folgenden gezeigt werden - bot dieses Konzept allen Parteien Anknüpfungspunkte für ihre jeweiligen parteipolitischen Positionen.

Die CDU/CSU-Fraktion, der es vornehmlich um die Förderung des ländlichen Raumes ging, unterstellte der SPD eine „sozialistische [...] Politik der ,konzentrierten Konzentration“ von Bevölkerung, Arbeitsplätzen und Infrastruktur“ (Jahn 1974: 7932), dem sie „das ordnungspolitische Leitbild der ,dezentralisierten Konzentration““ (ebd.) entgegensetze. Der 
CDU/CSU-Fraktion erschien dieses Konzept als alleinige Option, die Lebensbedingungen in den ländlichen Räumen zu verbessern:

„Die CDU/CSU bejaht Konzentration in der Fläche als einzige Möglichkeit, städtischen Lebensverhältnissen gleichwertige Umstände auch im ländlichen Raum zu schaffen. Das soll aber nicht heißen, daß nur in den zentralen Orten gebaut werden soll. Wir wollen angemessene Entwicklungs- und Siedlungstätigkeit auch in geeigneten kleineren Orten und Dörfern.“ (Jahn 1976: 16749; Herv. i. O.)

Dieses Ziel war mit einer konservativen Stoßrichtung verbunden, die zum Beispiel daran deutlich wird, dass aus Sicht der CDU/CSU-Fraktion „die Gemeinschaft in dem ländlichen Raum in der Fülle der Klein- und Mittelstädte“ (Warnke 1974: 7960) bestehe. Dennoch wollte die CDU/CSU-Fraktion eine „flächenabdeckende Raumordnungspolitik“ (CDU/CSU 1978: 2, Pack 1978: 3627). Dies war in ihrer Argumentation kein Widerspruch, denn dieser „konzentrierte Mitteleinsatz“ (Jahn 1977: 8) müsse „den ländlichen Raum miteinbeziehen“(ebd.). Die Attraktivität des Konzeptes der dezentralen Konzentration bestand für Konservative gerade darin, eine Politik für die ländlichen Räume als Orte des ,gemeinschaftlichen' Lebens zu betreiben.

Die SPD, deren Wähler_innen hauptsächlich in den Verdichtungsräumen ansässig waren, begründete die Forderung nach der ,dezentralisierte[n] Konzentration" (Immer 1975b: 4; Herv. i. O.) mit vier Argumenten. Erstens sei nur so ein Abbau der großräumigen Disparitäten möglich (vgl. Vogel 1973: 49). Die raumordnungspolitischen Mittel müssten zweitens effizient eingesetzt werden - schließlich wolle man „effektiv helfen“ (Immer 1978: 8629). Drittens sei der wirtschaftliche Konzentrationsprozess zu unterstützen, weil „die Industrialisierung in bestimmten Bereichen die Entleerung der Gemeinden doch nicht verhindern werde“ (Immer 1973: 43). Außerdem sei viertens die Schwerpunktbildung notwendig, um die Verdichtungsräume zu entlasten (vgl. Haack 1975: 23). Die SPD präferierte das Konzept der dezentralen Konzentration also, weil es ihr ermöglichte, die wirtschaftsräumliche Dynamik zentralstaatlich zu unterstützen und zu lenken und - nebenbei ihre Wähler_innen in den Städten zu fördern.

Die FDP präferierte - was für eine liberale Partei wenig verwunderlich ist - die dezentralisierte Konzentration zur Sicherstellung der „Chancengleichheit im räumlichen Sinn“ (Böger 1976: 16755). Für sie war die dezentrale Konzentration unerlässlich, um „die Attraktivität größerer Verdichtungsräume, dievon der Bevölkerung offensichtlich gewünscht wird, [...] in die heute dünn besiedelten ländlichen Räume hineinzutragen“(Engelhard 1974: 7949).

Auch die Bundesregierung befürwortete die „dezentral konzentrierte Entwicklung“ (1975a), um die großräumigen Disparitäten in der Bundesrepublik zu bearbeiten. Die Raumordnungspolitik mittels derer dies erreicht werden sollte, beinhaltete die Ausweisung von Entwicklungszentren und -achsen (vgl. Beirat für Raumordnung 1972a: 157ff.). Aufgrund des begrenzten Entwicklungspotentials sollte dadurch eine „konsequente Standortkonzentration und Bündelung raumwirksamer Fördermaßnahmen“ (Bundesregierung 1975b: 39) erfolgen. Man erhoffte sich zum einen, mithilfe der Entwicklungszentren und -achsen Wachstumsimpulse in strukturschwachen Gebieten auszulösen (vgl.ebd. 9).Zum anderen sollten dieVerdichtungsräume 
entlastet und „weit in das Umland ausstrahlende Impulse für das gesamte Bundesgebiet" (Bundesregierung 1974: 2) gesetzt werden. Obwohl dieses Konzept, da es für alle Parteien Anknüpfungspunkte bot, grundsätzlich nicht umstritten war, gab es im untersuchten raumordnungspolitischen Diskurs Auseinandersetzungen um die Ausweisung von Entwicklungszentren.

Der Streit entbrannte an der Mindestbevölkerungszahl von Entwicklungszentren, die im Bundesraumordnungsprogramm festgelegt werden sollte. Hintergrund dieses Disputes war ein Kompetenzstreit zwischen Bund und Ländern. Die Länder hatten bis in die 1970er-Jahre zentrale Orte ausgewiesen. Sie empfanden den Versuch des Bundes, die Entwicklungszentren und -achsen als neues „Grundgerüst der Raumordnungspolitik des Bundes und der Länder auszuweisen“ (Bundesregierung 1972: 14), als Eingriff in ihren Kompetenzbereich. Aus Sicht des Bundes bot die Ausweisung von Entwicklungszentren und -achsen die Möglichkeit, die eigenen Kompetenzen bezüglich Raumordnung auszuweiten und die räumliche Entwicklung in der Bundesrepublik stärker beeinflussen zu können. Der Bund wollte „nur eine relativ geringe Anzahl von Entwicklungsschwerpunkten“ (ebd.: 77) fördern. Die Länder hingegen wollten eine möglichst große Zahl an „förderungsfähigen“ (ebd.) Entwicklungsschwerpunkten in das Programm aufnehmen, ohne deren Anzahl zu begrenzen. Einigkeit bestand zwischen Bund und Ländern dahingehend, dass „Entwicklungsschwerpunkte eine Mindestbevölkerung von 40 ooo Einwohnern“ (ebd.) aufweisen sollten. Auch eine Ausnahmeregelung wurde diskutiert: Der Bund wollte Ausnahmen nur bis 20.00o Einwohner_innen zulassen, die Länder wollten sich hingegen auf keine konkrete Zahl festlegen lassen (ebd.). Letztlich konnten sich die Länder durchsetzen, sodass im Bundesraumordnungsprogramm keine konkrete Zielgröße genannt wurde (vgl. Bundesregierung 1975b: 37ff).

Auch in den untersuchten Bundestagsdebatten äußerten sich die Parteien zu diesem Konfliktpunkt. Aus Sicht der CDU/CSU-Fraktion war die vom Bund vorgeschlagene Mindesteinwohner_innenzahl eine Konzentrationspolitik, „die zu weit gehe“ (Jahn 1973: 31). Dies sei eine „Verödungspolitik für die ländlichen Räume“ (Jahn 1974: 7931) und führe in Bayern dazu, dass „70 der bestehenden 114 Schwerpunkte eliminiert“ (Warnke 1973: 34) würden. Die SPD wollte den Forderungen der CDU/CSU-Fraktion, die als „Gießkannenprinzip“ (Sperling 1978: 8634) abgekanzelt wurden, jedoch nicht weiter entgegenkommen. Solch ein „engmaschiges Netz“ (Immer 1975c: 15) könne die SPD „raumordnungspolitisch nicht akzeptieren“ (ebd.). An dieser stärker konzeptionellen Frage wird deutlich, dass die Parteien bestrebt waren, durch raumordnungspolitische Strategien ihr jeweiliges Wähler_innenklientel zu bedienen, sich damit allerdings eines Konfliktfeldes annahmen, das aufgrund eines Kompetenzstreites zwischen Bund und Ländern entstanden war.

Bis auf die Einwohner_innenzahl von Entwicklungszentren war das Konzept der dezentralen Konzentration nicht umkämpft. Die Parteien konnten ihre jeweiligen Vorstellungen mit dem Konzept aus zwei Gründen verbinden: Erstens bot das Konzept Anknüpfungspunkte für alle Parteien. Die CDU/CSU-Fraktion konnte damit die Förderung ländlicher Räume und Vergemeinschaftung einfordern. Der SPD ermöglichte es, die Konzentrationsprozesse zu unterstützen und damit die eigenen Wähler_innen in den Verdichtungsräumen. Für die FDP war die dezentrale Konzentration ein 
Mittel, um die freiheitliche Entwicklung auch in ländliche Räume zu tragen. Diese jeweiligen parteipolitischen Anknüpfungspunkte bot das Konzept der dezentralen Konzentration zweitens aufgrund der real stattfindenden räumlichen Entwicklung, die gerade die Form der dezentralen Konzentration angenommen hatte. Hinzu kommt, dass das Konzept zwar in raumordnungspolitische Programme beziehungsweise Pläne des Bundes aufgenommen wurde, diese aber für die Landesplanung und Bundesfachpolitiken kaum Bindungswirkung hatten (siehe Abschnitt 1).

\section{Fazit}

Das Wirtschaftswachstum vollzog sich in der Bundesrepublik Deutschland in den 1970er- Jahren räumlich in Form der dezentralen Konzentration. Aufgrund des Arbeitskräftemangels wurden jenseits der Verdichtungsgebiete sogenannte verlängerte Werkbänke eröffnet. Auf diese Weise sollte das Wirtschaftswachstum nicht ins Stocken geraten und Entwicklungsprozesse räumlich gestreut werden, auch wenn sich Konzentrationsprozesse innerregional fortsetzen. Darauf reagierte die Raumordnung mit der Strategie der dezentralen Konzentration, die mit dem Konzept der Entwicklungszentren und -achsen unterlegt wurde. Insofern protegierte die Raumordnungspolitik den real stattfindenden räumlichen Entwicklungsprozess zur Unterstützung der gesamtwirtschaftlichen Entwicklung und vollzog die reale regionale Entwicklung - die gerade die Form der dezentralen Konzentration angenommen hatte - lediglich nach.

Anders als häufig proklamiert (vgl. zum Beispiel Leendertz 2008: 363) war die Raumordnungspolitik damit nicht explizit darum bemüht, eine ausgeglichene Raumstruktur herzustellen. Die dezentrale Konzentration wirkte zwar aus einer großräumigen Perspektive räumlich ausgleichend, innerregional hingegen beförderte diese Entwicklungsstrategie die Disparitäten. Das Ziel war es vielmehr, das wirtschaftliche Wachstum zu unterstützen und in den 1970er-Jahren schien das Konzept der dezentralen Konzentration das geeignete regionalpolitische Mittel zu sein. Die Raumordnung fokussierte sich folglich allein auf großräumige Disparitäten, während innerregionale Unterschiede weniger wichtig wurden.

Die raumordnungspolitische Verdichtung der politischen Kräfteverhältnisse zu dieser kohärenten raumordnungspolitischen Strategie ermöglichte es, das Wirtschaftswachstum zu unterstützen. Die dezentrale Konzentration hatte aber noch ein zweites wichtiges Fundament: Durch sie konnten die Parteien - aufgrund der real stattfindenden räumlichen Entwicklung - ihre eigenen parteipolitischen Interessen durchsetzen. Der CDU/CSU-Fraktion galt die dezentrale Konzentration zum einen als Mittel, um die ländlichen Räume - und damit ihre Wähler_innen - zu fördern. Zum anderen konnte sie dadurch großstadtfeindliche Ressentiments bedienen. Die SPD verband mit der dezentralen Konzentration die Förderung städtischer Lebensweisen sowohl in den Agglomerationsräumen als auch in den ländlichen Regionen. Auch die SPD konnte damit ihre Wähler_innen - die großstädtischen Arbeiter_innen - protegieren. Für die FDP war die dezentrale Konzentration eine Möglichkeit, die Idee der freiheitlichen Entwicklung auch in ländliche und strukturschwache Regionen zu tragen. 
Die Methode der historisch-materialistischen Politikanalyse ermöglichte es, diesen Verdichtungsprozess der politischen Kräfteverhältnisse im Staat zu einer kohärenten raumordnungspolitischen Strategie aufzuzeigen, die den Notwendigkeiten des Wirtschaftswachstums gerecht wurde. Aufgrund der Kontextanalyse war es möglich, die raumordnungspolitische Strategie der dezentralen Konzentration als Strategie zu dechiffrieren, mittels derer das wirtschaftliche Wachstum unterstützt und folglich die - sich ohnehin vollziehende - räumliche Entwicklung protegiert wurde.

Abschließend sollen noch zwei Möglichkeiten zur Weiterentwicklung der historisch-materialistischen Politikanalyse benannt werden: Um der Gefahr zu entgehen, sich in kontextuellen Entwicklungen zu verlieren, müssen die Forschenden klar bestimmen, welcher Kontext für ihre Analyse relevant ist. Deshalb wurden in diesem Aufsatz - neben dem ökonomischen und politischen Kontext - insbesondere die relevanten räumlichen Entwicklungen herausgearbeitet. Weiterhin gestaltet sich die Operationalisierung der Akteurs- und Prozessanalyse in der Forschungspraxis relativ schwierig, da die vorgestellten Analyseschritte mehr eine Heuristik darstellen. Zur Lösung dieser Problematik wurde die Methode der critical discourse analysis gewählt, um die beiden Analyseschritte empirisch zu operationalisieren.

\section{Endnoten}

[1] Der Begriff der Verdichtung wird in der raumplanerischen/raumordnerischen Literatur für die bauliche Verdichtung von Räumen verwendet. Der Terminus der Verdichtung der gesellschaftlichen Kräfteverhältnisse stammt hingegen aus der materialistischen Staatstheorie. Diese begriffliche Nähe ist in diesem Aufsatz nicht vermeidbar, da die Erkenntnisse der materialistischen Staatstheorie für das Verständnis der Raumordnungspolitik genutzt werden. Der Begriff der Verdichtung im raumplanerischen Sinne wird hier jedoch nur für die Raumkategorie der Verdichtungsräume verwendet.

[2] Federführend war hier insbesondere die Forschungsgruppe <Staatsprojekt Europa $>$ (2014).

[3] Zu nennen wäre hier beispielsweise das Wirtschaftsministerium im Rahmen der Gemeinschaftsaufgabe Verbesserung der regionalen Wirtschaftsstruktur.

[4] Der Begriff der dezentralen Konzentration wird in der raumplanerischen und raumordnerischen Literatur für eine auf dezentrale Entwicklung setzende, dabei jedoch innerregionale Konzentration befördernde raumordnungspolitische Handlungsstrategie benutzt. Werner Väth (1980) argumentiert, dass die materielle Grundlage dieses raumordnungspolitischen Handlungspostulates die räumliche Entwicklung gewesen sei, die die Form der dezentralen Konzentration angenommen habe. Insofern, so Väths Argument, habe die Raumordnungspolitik der 1970er-Jahre die räumliche Entwicklung der Zeit nachvollzogen und nicht entgegen gewirkt.

\section{Autor_innen}

Michael Mießner forscht zu den Themen Raumordnungspolitik, regionale Entwicklung, Wohnungspolitik sowie politics of scale und ländlicher Entwicklung. michael.miessner@geo.uni-goettingen.de 


\section{Literatur}

Abelshauser, Werner (2004): Deutsche Wirtschaftsgeschichte von 1945 bis zur Gegenwart. München: Beck.

Altvater, Elmar / Hübner, Kurt (1988): Das Geld einer mittleren Hegemonialmacht. Ein kleiner Streifzug durch die ökonomische Geschichte der BRD. In: PROKLA. Probleme des Klassenkampfs - Zeitschrift für politische Ökonomie und sozialistische Politik 18/4, 6-36.

Beirat für Raumordnung (1972a): Empfehlungen des Beirats für Raumordnung. a) Zielsystem für die räumliche Entwicklung der Bundesrepublik Deutschland (28. Oktober 1971). In: Raumordnungsbericht 1972. Bonn: Verlag Dr. Hans Heger, 154-161.

Beirat für Raumordnung (1972b): Empfehlungen des Beirats für Raumordnung. b) Zielsystem zur räumlichen Ordnung und Entwicklung der Verdichtungsräume in der Bundesrepublik Deutschland (14. September 1972). In: Raumordnungsbericht 1972. Bonn: Verlag Dr. Hans Heger, 162-172.

Blotevogel, Hans H. (2011): Raumordnung im westlichen Deutschland 1945 bis 1990. In: Akademie für Raumforschung und Landesplanung (Hg.), Grundriss der Raumordnung und Raumentwicklung. Hannover: Verlag der ARL, 115-168.

BMWSR (Bundesminister für Wohnungswesen, Städtebau und Raumordnung) (1963): Erster Raumordnungsbericht. Bonn: Bonner Universitäts-Buchdruckerei.

Böger, Rolf (1976): Redebeitrag. In: 239. Sitzung des Deutschen Bundestages, Bonn, Freitag, den 7. Mai 1976. 7. Wahlperiode.

Brand, Ulrich (2013): State, context and correspondence. Contours of a cistorical-materialist policy analysis. In: Österreichische Zeitschrift für Politikwissenschaft 42/4, 425-442.

Brenner, Neil (1997): State territorial restructuring and the production of spatial scale. Urban and regional planning in the federal republic of Germany, 1960-1990. In: Political Geography 16/4, 273-306.

Brenner, Neil (2003): 'Glocalisation' as a state spatial strategy. Urban entrepreneurialism and the new politics of uneven development in western Europe. In: Jamie Peck / Henry Wai-Chung Yeung (Hg.), Remaking the Global Economy. Economic-geographical Perspectives. London u. a.: SAGE, 197-215.

Brenner, Neil (2004): New State Spaces. Urban Governance and the Rescaling of Statehood. Oxford u. a.: Oxford University Press.

Buckel, Sonja / Georgi, Fabian / Kannankulam, John / Wissel, Jens (2014): Theorie, Methoden und Analysen kritischer Europaforschung. In: Forschungsgruppe <Staatsprojekt Europa> (Hg.), Kämpfe um Migrationspolitik. Theorie, Methode und Analysen kritischer Europaforschung. Bielefeld: Transcript, 15-84.

Bundesregierung (Bundesregierung der Bundesrepublik Deutschland) (1966): Raumordnungsbericht 1966. Bonn : Bonner Universitäts-Buchdruckerei.

Bundesregierung (Bundesregierung der Bundesrepublik Deutschland) (1972): Raumordnungsbericht 1972. Bonn: Verlag Dr. Hans Heger.

Bundesregierung (Bundesregierung der Bundesrepublik Deutschland) (1974): Antwort auf die Große Anfrage der Abgeordneten Dr. Jahn (Münster), Dr. Schneider, Dr. Waffenschmidt, Dr. Warnke, Sick, Dr. Gruhl, Orgaß und Genossen und der Fraktion der CDU/CSU, Drucksache 7/1417 betr. Raumordnung. Bonn: Bonner Univ.-Buchdruckerei.

Bundesregierung (Bundesregierung der Bundesrepublik Deutschland) (1975a): Raumordnungsbericht 1974. Bonn: Bonner Universitäts-Buchdruckerei.

Bundesregierung (Bundesregierung der Bundesrepublik Deutschland) (1975b): Raumordnungsprogramm für die großräumige Entwicklung des Bundesgebietes (Bundesraumordnungsprogramm). Bonn: Bonner Universitäts-Buchdruckerei.

CDU/CSU (1978): Antrag der Abgeordneten Frau Pack, Dr. Dollinger, Dr. Jahn (Münster), Dr. Schneider, Dr. Möller, Sauter (Epfendorf), Sick, Dr. Ritz, Dr. Waffenschmidt, Nordlohne, Francke (Hamburg), Kolb, Niegel, Eymer (Lübeck), Dr. van Aerssen, Dr. Freiher Spies von Büllesheim, Burger, Dr. Warnke, Dr. George, Schedl, Dr. Unland, Frau Hoffmann (Hoya), Milz, Dr. Jobst, Feinendegen, Geisenhofer, Biehle, Neuhaus, Müller (Berlin), Susset und der Fraktion der CDU/CSU. Raumordnung. Bonn: Bonner Universitäts-Buchdruckerei.

Durner, Wolfgang / Greiving, Stefan / Reitzig, Frank (2011): Rechtlicher und institutioneller Rahmen der Raumplanung. In: Akademie für Raumforschung und Landesplanung (Hg.), Grundriss der Raumordnung und Raumentwicklung. Hannover: Verlag der ARL, 379-433.

Engelhard, Hans Arnold (1974): Redebeitrag. In: 119. Sitzung des Deutschen Bundestages, Bonn, Donnerstag, den 26. September 1974. 7. Wahlperiode. Bonn: Bonner Universitäts-Buchdruckerei. 
Engelhard, Hans Arnold (1978): Redebeitrag. In: 109. Sitzung des Deutschen Bundestages, Bonn, Donnerstag, den 5. Oktober 1978. 8. Wahlperiode. Bonn: Bonner Universitäts-Buchdruckerei.

Fairclough, Norman (2010): Critical Discourse Analysis. The Critical Study of Language. Harlow: Longman.

Fairclough, Norman / Pardoe, Simon / Szerszynski, Bronislaw (2010): Critical discourse analysis and citizenship. In: Norman Fairclough (Hg.), Critical Discourse Analysis. The Critical Study of Language. Harlow: Longman, 412-436.

Forschungsgruppe <Staatsprojekt Europa> (Hg.) (2014): Kämpfe um Migrationspolitik. Theorie, Methode und Analysen kritischer Europaforschung. Bielefeld: Transcript.

Friedrichs, Jürgen / Häußermann, Hartmut / Siebel, Walter (Hg.) (1986): Süd-Nord-Gefälle in der Bundesrepublik? Sozialwissenschaftliche Analysen. Opladen: Westdeutscher Verlag.

Gatzweiler, Hans Peter / Runge, Ludwig (1978): Regionale Disparitäten im Bundesgebiet. Erste Ergebnisse aus der laufenden Raumbeobachtung. In: Informationen zur Raumentwicklung 8/9, 669-693.

Haack, Dieter (1975): Redebeitrag. In: Kurzprotokoll, 65. Sitzung des Ausschusses für Raumordnung, Bauwesen und Städtebau am Mittwoch, dem 22. Oktober 1975.

Harvey, David (1981): The spatial fix - Hegel, von Thünen, and Marx. In: Antipode 13/3, 1-12.

Harvey, David (2004): Die Geographie des „neuen“ Imperialismus: Akkumulation durch Enteignung. In: Christian Zeller (Hg.), Die globale Enteignungsökonomie. Münster: Westfälisches Dampfboot, 183-215.

Harvey, David (2006): The Limits to Capital. London: Verso.

Heeg, Susanne (2001): Politische Regulation des Raums. Metropolen, Regionen, Nationalstaat. Berlin: Edition Sigma.

Hirsch, Joachim (1994): Politische Form, politische Institutionen und Staat. In: Josef Esser / Christoph Görg / Joachim Hirsch (Hg.), Politik, Institutionen und Staat. Zur Kritik der Regulationstheorie. Hamburg: VSA-Verlag, 157-211.

Hirsch, Joachim / Kannankulam, John (2006): Poulantzas und Formanalyse. Zum Verhältnis zweier Ansätze materialistischer Staatstheorie. In: Lars Bretthauer / Alexander Gallas / John Kannankulam / Ingo Stützle (Hg.), Poulantzas lesen. Zur Aktualität marxistischer Staatstheorie. Hamburg: VSA-Verlag, 65-81.

Holgersen, Ståle (2013): Spatial planning as condensation of social relations: A dialectical approach. In: Planning Theory 14/1, 5-22.

Immer, Klaus (1973): Redebeitrag. In: Stenographisches Protokoll, 7. Sitzung des Ausschusses für Raumordnung, Bauwesen und Städtebau am Mittwoch, dem 6. Juni 1973.

Immer, Klaus (1975a): Bundesraumordnungsbericht 1974, Bundestagsdrucksache 7/3582; hier: Bericht für die Beratung im Bundestagsausschuß für Raumordnung, Bauwesen und Städtebau. Anlage 3 zu: Kurzprotokoll, 65. Sitzung des Ausschusses für Raumordnung, Bauwesen und Städtebau am Mittwoch, dem 22. Oktober 1975. Bonn: Bonner Universitäts-Buchdruckerei.

Immer, Klaus (1975b): Raumordnungsprogramm für die großräumige Entwicklung des Bundesgebietes; hier: Bericht für die Beratung im Bundestagsausschuß für Raumordnung, Bauwesen und Städtebau (Bundesraumordnungsprogramm), Bundestagsdrucksache 7/3584. Anlage zu: Kurzprotokoll, 65. Sitzung des Ausschusses für Raumordnung, Bauwesen und Städtebau am Mittwoch, dem 22. Oktober 1975. Bonn.

Immer, Klaus (1975c): Redebeitrag. In: Kurzprotokoll, 65. Sitzung des Ausschusses für Raumordnung, Bauwesen und Städtebau am Mittwoch, dem 22. Oktober 1975.

Immer, Klaus (1978): Redebeitrag. In: 109. Sitzung des Deutschen Bundestages, Bonn, Donnerstag, den 5. Oktober 1978. 8. Wahlperiode. Bonn.

Jacob, Joachim (1979): Regionalpolitik und räumliche Disparitäten in der Bundesrepublik. Zur Kritik der Regionalförderung in den Deglomerationsräumen. Frankfurt/ Main: R.G. Fischer.

Jahn, Friedrich-Adolf (1973): Redebeitrag. In: Stenographisches Protokoll, 7. Sitzung des Ausschusses für Raumordnung, Bauwesen und Städtebau am Mittwoch, dem 6. Juni 1973. Bonn.

Jahn, Friedrich-Adolf (1974): Redebeitrag. In: 119. Sitzung des Deutschen Bundestages, Bonn, Donnerstag, den 26. September 1974. 7. Wahlperiode. Bonn: Bonner Universitäts-Buchdruckerei. 
Jahn, Friedrich-Adolf (1976): Redebeitrag. In: 239. Sitzung des Deutschen Bundestages, Bonn, Freitag, den 7. Mai 1976. 7. Wahlperiode. Bonn: Bonner Universitäts-Buchdruckerei.

Jahn, Friedrich-Adolf (1977): Redebeitrag. In: Kurzprotokoll der 14. Sitzung des (15.) Ausschusses für Raumordnung, Bauwesen und Städtebau, Mittwoch, 29. Juni 1977. Bonn.

Jessop, Bob (1990): State Theory. Putting the Capitalist State in its Place. Pennsylvania: Pennsylvania State University Press.

Jessop, Bob (2008): State Power. A Strategic-Relational Approach. Cambridge u. a.: Polity-Press.

Kannankulam, John (2008): Autoritärer Etatismus im Neoliberalismus. Zur Staatstheorie von Nicos Poulantzas. Hamburg: VSA-Verlag.

Kannankulam, John / Georgi, Fabian (2012): Die Europäische Integration als materielle Verdichtung von Kräfteverhältnissen. Hegemonieprojekte im Kampf um das ‘Staatsprojekt Europa'. Marburg.

Kannankulam, John / Georgi, Fabian (2014): Varieties of capitalism or varieties of relationships of forces? Outlines of a historical materialist policy analysis. In: Capital \& Class 38/1, 59-71.

Krumbein, Wolfgang / Frieling, Hans-Dieter von / Kröcher, Uwe / Sträter, Detlev (2008): Zur Historie einer kritischen Regionalwissenschaft. Auch eine Einleitung. In: Wolfgang Krumbein / Hans-Dieter von Frieling / Uwe Kröcher / Detlev Sträter (Hg.), Kritische Regionalwissenschaft. Gesellschaft, Politik, Raum - Theorien und Konzepte im Überblick. Münster: Westfälisches Dampfboot, 7-40.

Leendertz, Ariane (2008): Ordnung schaffen. Deutsche Raumplanung im 20. Jahrhundert. Göttingen: Wallstein.

Marx, Karl (1962): Das Kapital. Kritik der politischen Ökonomie. Berlin: Dietz.

Mießner, Michael (2009): Der raumordnungspolitische Umgang mit regionalen Disparitäten in der BRD am Beispiel der Raumordnungsberichte. Unveröffentlichte Diplomarbeit. Göttingen.

Mießner, Michael (2015): Die Raumordnungspolitik der Bundesrepublik in der Nachkriegszeit. Raumordnungspolitische Konzepte als konservativer Ballast oder Antwort auf drängende Probleme der räumlichen Entwicklung? In: Wendelin Strubelt / Detlef Briesen (Hg.), Raumplanung nach 1945. Kontinuitäten und Neuanfänge in der Bundesrepublik Deutschland. Frankfurt am Main: Campus, 197-223.

Nordlohne, Franz-Josef (1973): Redebeitrag. In: Stenographisches Protokoll, 7. Sitzung des Ausschusses für Raumordnung, Bauwesen und Städtebau am Mittwoch, dem 6. Juni 1973. Bonn.

Pack, Doris (1978): Redebeitrag. In: 109. Sitzung, Bonn, Donnerstag, den 5. Oktober 1978. 8. Wahlperiode. Bonn: Bonner Universitäts-Buchdruckerei.

Paschukanis, Eugen (1929): Allgemeine Rechtslehre und Marxismus. Versuch einer Kritik der juristischen Grundbegriffe. Wien u. a.: Verlag für Literatur und Politik.

Polster, Werner / Voy, Klaus (1991): Von der politischen Regulierung zur Selbstregulierung der Märkte - Die Entwicklung von Wirtschafts- und Ordnungspolitik in der Bundesrepublik. In: Klaus Voy / Werner Polster / Claus Thomasberger (Hg.), Marktwirtschaft und politische Regulierung. Beiträge zur Wirtschafts- und Gesellschaftsgeschichte der Bundesrepublik Deutschland (1949-1989). Marburg: Metropolis, 169-226.

Poulantzas, Nicos (2002): Staatstheorie. Politischer Überbau, Ideologie, autoritärer Etatismus. Hamburg: VSA-Verlag.

Prollius, Michael von (2006): Deutsche Wirtschaftsgeschichte nach 1945. Göttingen: Vandenhoeck \& Ruprecht.

SVR (Sachverständigenrat zur Begutachtung der gesamtwirtschaftlichen Entwicklung) (1965): Jahresgutachten 1965/66. Stabilisierung ohne Stagnation. Stuttgart.

SVR (Sachverständigenrat zur Begutachtung der gesamtwirtschaftlichen Entwicklung) (1967): Jahresgutachten 1967/68 des Sachverständigenrates zur Begutachten der gesamtwirtschaftlichen Entwicklung. Stabilität im Wachstum. Stuttgart.

SVR (Sachverständigenrat zur Begutachtung der gesamtwirtschaftlichen Entwicklung) (1975): Jahresgutachten 1975 des Sachverständigenrates zur Begutachten der gesamtwirtschaftlichen Entwicklung. Vor dem Aufschwung. Bonn: Bonner Universitäts-Buchdruckerei.

SVR (Sachverständigenrat zur Begutachtung der gesamtwirtschaftlichen Entwicklung) (1976): Jahresgutachten 1976/77 des Sachverständigenrates zur Begutachtung der gesamtwirtschaftlichen Entwicklung. Zeit zum Investieren. Bonn: Bonner Universitäts-Buchdruckerei. 
SVR (Sachverständigenrat zur Begutachtung der gesamtwirtschaftlichen Entwicklung) (1978): Jahresgutachten 1978/79 des Sachverständigenrates zur Begutachtung der gesamtwirtschaftlichen Entwicklung. Wachstum und Währung. Bonn: Bonner Universitäts-Buchdruckerei.

Sauter, Franz (1978): Redebeitrag. In: 109. Sitzung des Deutschen Bundestages, Bonn, Donnerstag, den 5. Oktober 1978. 8. Wahlperiode. Bonn: Bonner Universitäts-Buchdruckerei.

Schiller, Karl (1964): Wirtschaftspolitik (1962). In: Karl Schiller (Hg.), Der Ökonom und die Gesellschaft. Das freiheitliche und das soziale Element in der modernen Wirtschaftspolitik. Stuttgart: Gustav Fischer Verlag, 63-90.

Schmitt-Vockenhausen, Hermann (1974): Redebeitrag. In: 119. Sitzung des Deutschen Bundestages, Bonn, Donnerstag, den 26. September 1974. 7. Wahlperiode. Bonn: : Bonner Universitäts-Buchdruckerei.

Sinz, Manfred (2005): Raumordnung / Raumordnungspolitik. In: Ernst-Hasso Ritter (Hg.), Handwörterbuch der Raumordnung. Hannover: ARL, 863-872.

Smith, Neil (1984): Uneven Development. Nature, Capital and the Production of Space. Oxford: Blackwell.

Sperling, Dietrich (1978): Redebeitrag. In: 109. Sitzung des Deutschen Bundestages, Bonn, Donnerstag, den 5. Oktober 1978. 8. Wahlperiode.

Väth, Werner (1979): Ökonomische Stagnation und Raumordnungspolitik. In: Wolfgang Brunder / Thomas Ellwein (Hg.), Raumordnung und staatliche Steuerungsfähigkeit. Opladen: Westdeutscher Verlag, 58-75.

Väth, Werner (1980): Raumplanung. Probleme der räumlichen Entwicklung und Raumordnungspolitik in der Bundesrepublik Deutschland. Königstein: Hain.

Vogel, Hans-Jochen (1973): Redebeitrag. In: Stenographisches Protokoll, 7. Sitzung des Ausschusses für Raumordnung, Bauwesen und Städtebau am Mittwoch, dem 6. Juni 1973. Bonn.

Warnke, Jürgen (1973): Redebeitrag. In: Stenographisches Protokoll, 7. Sitzung des Ausschusses für Raumordnung, Bauwesen und Städtebau am Mittwoch, dem 6. Juni 1973. Bonn.

Warnke, Jürgen (1974): Redebeitrag. In: 119. Sitzung des Deutschen Bundestages, Bonn, Donnerstag, den 26. September 1974. 7. Wahlperiode. Bonn: Bonner Universitäts-Buchdruckerei.

Wissel, Jens (2015): Staatsprojekt EUropa: Grundzüge einer materialistischen Theorie der Europäischen Union. Münster: Westfälisches Dampfboot.

Wissel, Jens / Wöhl, Stefanie (2008): Staatstheorie vor neuen Herausforderungen. Einleitung. In: Jens Wissel / Stefanie Wöhl (Hg.), Staatstheorie vor neuen Herausforderungen: Analyse und Kritik. Münster: Westfälisches Dampfboot, 7-22.

Wodak, Ruth / Meyer, Michael (Hg.) (2001): Methods of Critical Discourse Analysis. London: SAGE.

\section{Spatial planning as condensation of political and societal forces}

The article analyses the condensation of political and societal forces concerning spatial planning in the Federal Republic of Germany in the 1970 s by using the historical-materialist policy analysis. Within these condensed political and societal forces uneven development should be processed by the concept of decentralized concentration through development centers and axes. Against the background of the actual uneven development in the 1970 - in the form of decentralized concentration - the different party members could connect their ideological and political ideas with the concept of decentralized concentration. In the end, the concept of decentralized concentration has led to a promotion of the already existing form of uneven development instead of preventing this development. 